(C) World Scientific Publishing Co. \& Operational Research Society of Singapore

\title{
NEAR-OPTIMAL MODIFIED BASE STOCK POLICIES FOR THE CAPACITATED INVENTORY PROBLEM WITH STOCHASTIC DEMAND AND FIXED COST
}

\author{
OKAN ÖRSAN ÖZENER* \\ Ozyegin University \\ Istanbul, Turkey \\ orsan.ozener@ozyegin.edu.tr \\ REFIK GÜLLÜ \\ Bogazici University \\ Istanbul, Turkey \\ refik.gullu@boun.edu.tr \\ NESIM ERKIP \\ Bilkent University \\ Ankara, Turkey \\ nesim@bilkent.edu.tr \\ Received 7 March 2013 \\ Revised 5 August 2013 \\ Accepted 24 October 2013 \\ Published 6 January 2014
}

\begin{abstract}
In this study, we investigate a single-item, periodic-review inventory problem where the production capacity is limited and unmet demand is backordered. We assume that customer demand in each period is a stationary, discrete random variable. Linear holding and backorder cost are charged per unit at the end of a period. In addition to the variable cost charged per unit ordered, a positive fixed ordering cost is incurred with each order given. The optimization criterion is the minimization of the expected cost per period over a planning horizon. We investigate the infinite horizon problem by modeling the problem as a discrete-time Markov chain. We propose a heuristic for the problem based on a particular solution of this stationary model, and conduct a computational study on a set of instances, providing insight on the performance of the heuristic.
\end{abstract}

Keywords: Inventory; stochastic processes; capacity restriction; fixed ordering cost.

\section{Introduction and Literature Review}

To reduce the costs and gain a competitive advantage, firms must improve the efficiency of their operations. Inventory management plays a key role in increasing

\footnotetext{
* Corresponding author
} 
efficiency. Matching supply and demand is a critical challenge and to become successful at it, firms must lower inventory-related costs while satisfying customer demand. Although the benefits are appealing, identifying the most effective inventory control policy may be challenging. There is a significant amount of work in the literature on characterizing the optimal inventory control policy under numerous settings. We refer the reader to Girlich and Chikán (2001), which provides a historical analysis of development of the mathematical tools for inventory modeling such as the ones in probability theory, stochastic processes, game theory and dynamic programming.

Even though inventory related decisions are made at a firm level, a broader view is often required as this process involves several firms in a supply chain. Several factors such as cost structure, capacity restrictions, lead times, length of the planning horizon contribute to the complexity of the inventory management activities. Thus, the most effective strategies for controlling inventories are difficult to identify. Hence if the target is to identify optimal policy structure, one may need to assume simpler environments. Well-defined structures are important learning tools and probable candidates to become building blocks for more complicated environments. The basic newsvendor problem is one such example.

When the newsvendor model is extended to multiple periods, the optimal ordering policy becomes a base stock policy. In a base stock policy, if the initial inventory position in any period is below a critical level $S$, enough should be ordered to bring it up to $S$, otherwise nothing should be ordered (Scarf, 1960).

When a positive fixed ordering cost $K$ is introduced, then optimal policy is an $(s, S)$ type policy (Scarf, 1960). In $(s, S)$ policy, if the inventory position falls below $s$, enough should be ordered to bring it up to $S$, otherwise nothing should be ordered.

When there is no fixed ordering cost, but a production capacity, then a modified base stock policy is optimal (Federgruen and Zipkin, 1986). In this policy, if the inventory position falls below $S$, enough should be ordered to bring it up to $S$; if this is not possible then the full capacity should be ordered.

When a fixed ordering cost and a finite upper bound on the order amount are both present in a single-item periodic inventory problem, the optimal ordering policy has not been fully identified. Wjingaard (1972) presents a three-period problem with deterministic demand where modified $(s, S)$ type policies are not optimal. In a modified $(s, S)$ policy, if the inventory level falls below $s$, enough should be ordered to bring the inventory position up to $S$ or as close as possible to $S$. Shaoxiang and Lambrecht (1996) attempt to characterize the optimal solution and suggest that optimal policy shows a pattern of $X-Y$ band structure. This structure can be described as follows: It is optimal to order the full capacity when the inventory level drops below $X$, and it is optimal to order nothing when the inventory level is above $Y$. Between $X$ and $Y$ the ordering pattern depends on the problem. Gallego and Scheller-Wolf (2000) extend the work of Shaoxiang and Lambrecht (1996) and partially characterize the optimal ordering policy. They suggest dividing the space into four regions and show that the optimal capacitated policy has an $(s, S)$-like 
structure, depending on the regions. In two of these regions, the optimal policy is completely specified, while in the other two regions, it is partially specified. Chan and Song (2003) provide an efficient algorithm to compute the optimal ordering policy parameters and show that it is enough to compute optimal ordering quantities for only a subset of inventory positions falling between $X$ and $Y$ bounds. Wijngaard and van Foreest (2010) consider a variation of the problem where the continuous production rate is finite and show that an $(s, S)$ type policy is optimal. In this setting, when the inventory level drops to $s$, a production is triggered (with a set-up cost) and continued until the inventory level reaches $S$. Finally, Shaoxiang (2004) proves that the length of the $X-Y$ band cannot be more than the capacity of a period. Shaoxiang (2004) also argues that the modified $(s, S)$ policy is not optimal for the infinite horizon problem (under discounted cost criterion), however, shows that the computational performance of the modified $(s, S)$ policy is generally reasonable. In summary, this problem have been investigated in the literature for more than 35 years, however, the optimal inventory policy structure has not been entirely characterized yet.

In this study, we do not attempt to further explore the structural properties of the problem. Rather, we intend to develop heuristic algorithms to generate nearoptimal solutions. We first model the infinite horizon problem as a discrete-time Markov chain (DTMC) under a certain ordering policy with a stationary threshold. Under this particular capacitated policy with an $(s, S)$-like structure, if the inventory drops below a certain threshold then we order/produce up to a certain level. If it is not possible to reach that order-up-to-level, we order/produce the full capacity. Finally, if the inventory level is above the threshold, we do not order/produce. A special case of this policy is called the "all-or-nothing" policy, which is discussed in related works such as Gallego and Toktay (2004) and Ozer and Wei (2004). Ozer and Wei (2004) note that in process industries such as oil and gas, sugar refining, and steel or aluminum hot roll pressing, all-or-nothing type policies are commonly observed due to high fixed costs of production. We apply this modified $(s, S)$ policy as a heuristic for the infinite planning horizon problem and compare its performance with the other heuristics, the all-or-nothing policy and the modified base stock policy, and finally with the optimal ordering policy computed with dynamic programming (DP).

The study is organized as follows: In Sec. 2, we give the formal statement and basic notation of the problem and provide some properties of the optimal order policy for this problem. In Sec. 3, we investigate the infinite horizon problem by defining the problem as a DTMC model. We develop a heuristic approach to the infinite horizon problem, namely the "best $(s, \Delta)$ policy" and evaluate the performance of this heuristic by comparing the results with two benchmark heuristics the "all-or-nothing policy" and the "modified base stock policy" and the DP solution. The results of this comparison are presented in Sec. 4. Finally, we provide concluding remarks in Sec. 5. 


\section{Description of the Problem}

We analyze a single-item, periodic-review production/inventory problem. We assume that demand in any period is a discrete random variable, which is independent and identically distributed from one period to another. Linear holding and backorder costs are charged per unit of inventory at the end of each period and a fixed ordering cost is associated with each order decision. Furthermore, the ordering quantity in any period is limited, with a positive capacity value. Without loss of generality, as in Chan and Song (2003), we assume that the production/order leadtime is zero. The objective is to minimize the expected cost per period over a finite or infinite horizon.

The sequence of events is as follows: At the beginning of each period, considering the inventory position value, decision of how much to produce is made. Because of the production capacity, it may not be possible to reach some inventory levels by ordering. If production occurs, other than a variable production cost per unit ordered, a fixed ordering cost is incurred for that period. The ordered amount arrives instantaneously, then demand is realized and satisfied with on-hand inventory. Unsatisfied demand is fully backordered, and holding and backorder costs are charged at the end of the period.

\subsection{Notation of the problem}

The following notation is used throughout this study:

$n$ : Period index.

$D_{n}$ : Non-negative random demand of period $n$ (when there are $n$ periods left in the planning horizon). Demand in any period is independent and identically distributed from period to period with a probability distribution $p_{r}$, i.e., $p_{r}$ : $\operatorname{Pr}(D=r), r=0,1, \ldots$

$C$ : Capacity of production, a positive integer. We assume $E[D]<C$ for ensuring long-run stationarity.

$v$ : Unit variable cost.

$b$ : Backorder cost per unit per period.

$h$ : Holding cost per unit per period.

$K$ : Fixed cost of ordering.

$x_{n}$ : Inventory position prior to placing any order in period $n$.

$y_{n}$ : Inventory position after placing an order, before demand is realized in period $n$.

Let $L(y)$ be the one period expected holding and backorder cost function with a given inventory position $y$ in period $n . L(y)$ can be expressed as;

$$
L(y)=h E\left[(y-D)^{+}\right]+b E\left[(D-y)^{+}\right] .
$$

$L(y)$ is a non-negative convex function and has a minimum point at a finite inventory position. Let $J_{n}(x)$ represent the expected optimal cost for an $n$-period horizon 
problem if the beginning inventory position is $x$. Let $G_{n}(y)$ represent the expected optimal cost for an $n$-period horizon problem if the inventory position after a production decision is $y$. Let $\delta(z)$ be an indicator function showing whether a fixed ordering cost is incurred or not $(\delta(z)=1$, when $z>0)$. $G_{n}(y)$ and $J_{n}(x)$ are as follows:

$$
\begin{aligned}
& G_{n}(y)=v y+L(y)+E\left[J_{n-1}\left(y-D_{n}\right)\right], \\
& J_{n}(x)=-v x+\min _{x \leq y \leq x+C}\left\{G_{n}(y)+K \delta(y-x)\right\} .
\end{aligned}
$$

We assume that at the end of the planning horizon, all leftover inventory is salvaged with zero cost/profit. In other words, $J_{0}(\cdot)=G_{0}(\cdot)=0$ for any inventory position value.

$S_{n}$ : Inventory position where the minimum of $G_{n}(y)$ is achieved in any period $n$. Global minimum of $G_{n}(y)$ may not be unique; in that case the minimum of these inventory levels is taken as $S_{n}$.

$s_{n}$ : Smallest inventory position where it is optimal not to order in period $n$.

\subsection{An illustrative example}

The optimal ordering policy of the single-period problem is a modified $(s, S)$ type policy. However, the single-period optimal policy cannot be extended to a multiperiod problem. We illustrate this phenomenon with an example. Even though the example is for the finite horizon problem for the sake of proven optimality of the order policy, it shows that the optimal order policy is not a simple monotone policy. In rest of the paper, however, we analyze the infinite horizon problem. Suppose that $h=1.0, b=15.0, K=55.0, v=1.0$, and $C=20$ and demand is equal to 8 with a probability of 0.95 and 9 with a probability of 0.05 . In Fig. 1(a), the graph of function $G_{7}(y)$ is presented. The function $G_{7}(y)$ has seven local minimum points at inventory values $8,17,20,24,40,48$, and 56 , and a global minimum point at 36. The local minimum points of $G_{n}(y)$ correspond to the combinations of possible demand values and the capacity. Because of the local minimum points the order amount function has an unusual pattern.

We observe from the order quantity versus inventory position graph (Fig. 1(b)) that the order quantity is not a non-increasing function of the inventory position. In fact, it is a combination of several modified $(s, S)$ order quantity functions. We observe from Fig. 1 that there exist several "kinks" in the curve of the optimal order quantity, as a function of inventory level. These "kinks" in the order amount function have resulted from the capacity constraint and the local minimum points. Function $G_{n}(y)$ is neither convex nor $K$-convex. Therefore, at a specific inventory position $y$, it may be optimal to order less than capacity to reach a local minimum point rather than full capacity, even though it is optimal to order full capacity at an inventory position greater than $y$. Because of this property of the order quantity function, it is difficult to determine the optimal policy for the multi-period problem. 


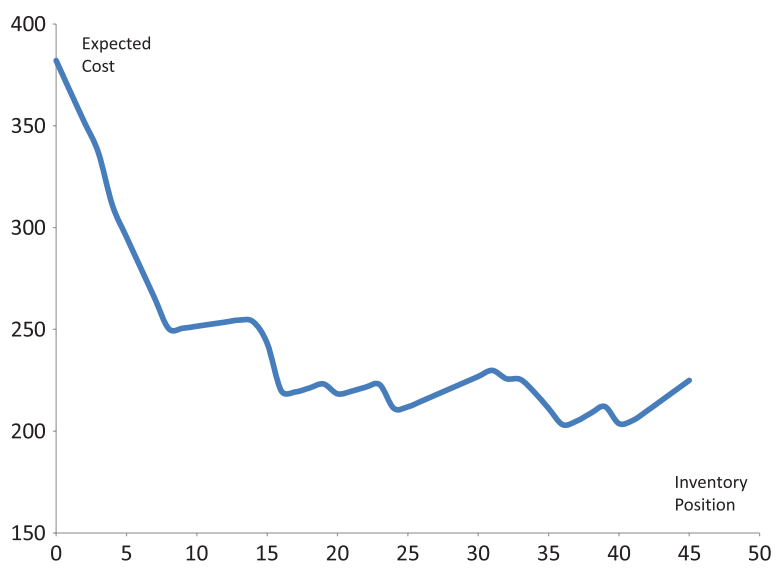

(a) Curve of $G_{7}(y)$

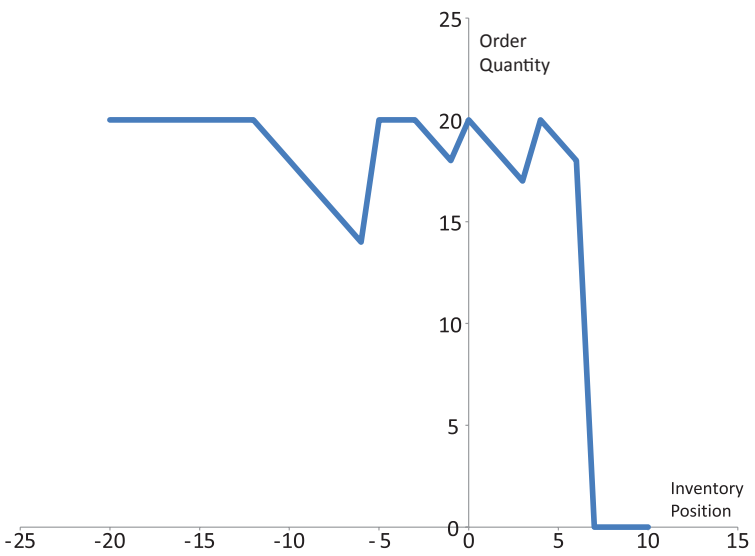

(b) Curve of optimal order quantity function

Fig. 1. Expected cost and order quantity functions of a seven-period-to-go problem with $h=1$, $b=15, K=55, v=1, C=20$, and $D=8$ with probability 0.95 , and $D=9$ with probability 0.05 .

In fact, for all the minimum points of the cost function, modified $(s, S)$ policies should be identified as in the single-period problem and then a combination of these modified $(s, S)$ policies will be the optimal order policy for the multi-period problem. Unfortunately, as the number of periods-to-go increases, the number of local minimum points also increases, and significantly, because the local minimum points are combinations of possible demand values and capacity. Hence, it is difficult to identify a general behavior of the order amount function and the optimal policy structure of the multi-period problem. However, it is apparent that the optimal policy for this problem structure will not be a simple monotone policy. 


\section{Infinite Horizon Problem}

\subsection{Analysis of the proposed policy}

In this section, we analyze the infinite horizon problem under average cost criterion and stationary demand/cost parameters. We apply a modified $(s, S)$ policy characterized by a threshold level as the ordering policy for this problem.

We model the infinite horizon problem for the general discrete demand case as a DTMC, by restricting the ordering policy to a modified $(s, S)$ policy with a threshold level. Our objective is to develop a well-performing policy that requires minimal computational effort and simple enough to implement in practice. To this end, we propose a capacitated policy with an $(s, S)$-like structure considering both the capacity restriction and fixed cost. Specifically, this policy operates with two parameters, the threshold level $s$ and a minimum positive order quantity $\Delta$. Because the maximum amount that can be ordered is $C, \Delta \in\{1,2, \ldots, C\}$. At the beginning of any period, if the inventory position is greater than or equal to $s$, then no order is placed. Otherwise, an order is placed to raise the inventory position as close as possible to $S=s-1+\Delta$. Note that $\Delta=1$ corresponds to the modified base stock policy with the base stock level equal to $s$, and $\Delta=C$ corresponds to the all-or-nothing policy. For brevity, we name this policy the $(s, \Delta)$ policy.

Generally speaking, when the fixed cost is relatively low, optimal policy becomes similar to the modified base stock policy, justifying even a low-quantity order if required. On the other hand, when the fixed cost is relatively high, it might be only economical to order at the capacity level. For all other cases, a different $(s, \Delta)$ policy might provide a good approximation to the optimal policy. Next, we show how the optimal values of two parameters are obtained.

We construct a DTMC for the problem under $(s, \Delta)$ policy and calculate the expected average cost per period for a given $(s, \Delta)$ using steady state distribution. Next, for a given $\Delta$, we show that optimal $s$ value is the smallest value that satisfies a critical ratio similar to the basic newsvendor model. Finally, we calculate the optimal $\Delta$ value based on the expected average cost per period and determine the best $(s, \Delta)$ policy. A pseudo-code of this approach is presented in Sec. 3.2 (Algorithm 3.1).

The following sequence of events takes place in any period: At the beginning of each period, depending on the inventory position, a decision to produce (order) may be made. That is, if the inventory position is below $s$, production occurs. Other than the variable production cost, a fixed ordering cost is incurred for that period. The ordered amount arrives instantaneously, raising the inventory position (as leadtime is zero, it is equivalent to the net inventory) by the produced (ordered) quantity. Then, demand is realized and satisfied with on-hand inventory. Unsatisfied demand is fully backordered, and corresponding holding and backorder costs are charged at the end of the period.

Let $x_{n+1}$ be the inventory level at the end of period $n$, and define $W_{n}=S-x_{n+1}$ as the shortfall from $S=s-1+\Delta$ at the end of the period. Let $U\left(W_{n}\right)$ be the amount ordered. Then, $(s, \Delta)$ policy prescribes that no order is placed at the 
beginning of period $n+1$ if $W_{n} \in\{0,1, \ldots, \Delta-1\}\left(U\left(W_{n}\right)=0\right)$, and an amount of $U\left(W_{n}\right)=\min \left\{W_{n}, C\right\}$ is placed whenever $W_{n} \in\{\Delta, \Delta+1, \ldots\}$. After the realization of demand $D_{n+1}$ in period $n+1, W_{n+1}$ is obtained as $W_{n+1}=W_{n}-U\left(W_{n}\right)+$ $D_{n+1}$ or

$$
W_{n+1}= \begin{cases}W_{n}+D_{n+1}, & W_{n} \in\{0,1, \ldots, \Delta-1\}, \\ D_{n+1}, & W_{n} \in\{\Delta, \ldots, C\}, \\ W_{n}-C+D_{n+1}, & W_{n} \in\{C+1, C+2, \ldots\} .\end{cases}
$$

We note that the evolution of the process $\left\{W_{n}, n=0,1, \ldots\right\}$ depends on $\Delta$, but does not depend on $s$. In other words, once the initial shortfall is given (which depends on $s$ ), the subsequent transitions are independent of $s$. Moreover, $W_{n}$ and $D_{n+1}$ are independent, thus it is clear that $\left\{W_{n}, n=0,1, \ldots\right\}$ is a DTMC on nonnegative integers for a given value of $\Delta$. Transition probabilities, $a_{i, j}=\operatorname{Pr}\left\{W_{n+1}=\right.$ $\left.j \mid W_{n}=i\right\}$, for this Markov chain can be obtained as:

$$
a_{i, j}= \begin{cases}0 & \text { for } i \in\{0,1, \ldots, \Delta-1\} \text { and } j<i, \\ p_{j-i} & \text { for } i \in\{0,1, \ldots, \Delta-1\} \text { and } j \geq i, \\ p_{j} & \text { for } i \in\{\Delta, \Delta+1, \ldots, C\} \text { and } j \geq 0, \\ 0 & \text { for } i \in\{C+1, C+2, \ldots\} \text { and } j<i-C, \\ p_{j+C-i} & \text { for } i \in\{C+1, C+2, \ldots\} \text { and } j \geq i-C .\end{cases}
$$

$\left\{W_{n}, n=0,1, \ldots\right\}$ has a stationary distribution $\Pi=\left\{\pi_{i}, i=0,1, \ldots\right\}$ provided that $E[D]=\sum_{r=0}^{\infty} p_{r} r<C$ (see Sec. 3 of Glynn (1989)). Unfortunately, determining $\Pi$ is not easy. We employ a state reduction algorithm described in Heyman and Sobel (1991) for numerically determining the steady state probabilities.

After determining the steady state probabilities, we formulate the expected average cost per period using these probabilities. Let $W$ be the random variable denoting the stationary shortfall with distribution $\Pi$ and let $\mathrm{AC}(s, \Delta)$ be the average expected cost of an $(s, \Delta)$ policy for a given $s$ and $\Delta$. Then,

$$
\begin{aligned}
\operatorname{AC}(s, \Delta)= & E\left[L(s+\Delta-1-W) 1_{\{W \in\{0,1, \ldots, \Delta-1\}\}}\right] \\
& +E\left[L(s+\Delta-1) 1_{\{W \in\{\Delta, \ldots, C\}\}}\right] \\
& +E\left[L(s+\Delta-1-W+C) 1_{\{W \in\{C+1, C+2, \ldots\}\}}\right] \\
& +\sum_{i=\Delta}^{\infty} \pi_{i}(K+v \min \{i, C\}),
\end{aligned}
$$

where $1_{\{A\}}$ takes a value 1 if $A$ is true, and takes 0 otherwise. Because $L(y)$ is convex in $y, \operatorname{AC}(s, \Delta)$ is convex in $s$ for a given $\Delta$. Therefore, the minimizer value of $s$ for a given $\Delta$ is obtained by

$$
s^{*}(\Delta)=\min \{s: \operatorname{AC}(s+1, \Delta)-\operatorname{AC}(s, \Delta) \geq 0\} .
$$


$L(y+1)-L(y)=-b+(h+b) F(y)$, where $F(y)=\operatorname{Pr}\{D \leq y\}$, thus we obtain that

$$
\begin{aligned}
\mathrm{AC}(s+1, \Delta)-\mathrm{AC}(s, \Delta)= & -b+(h+b) E\left[F(s+\Delta-1-W) 1_{\{W \in\{0,1, \ldots, \Delta-1\}\}}\right] \\
& +(h+b) E\left[F(s+\Delta-1) 1_{\{W \in\{\Delta, \ldots, C\}\}}\right] \\
& +(h+b) E\left[F(s+\Delta-1-W+C) 1_{\{W \in\{C+1, C+2, \ldots\}\}}\right],
\end{aligned}
$$

which can be rewritten as

$$
\begin{aligned}
\mathrm{AC}(s+1, \Delta)-\mathrm{AC}(s, \Delta)= & -b+(h+b)\left\{\sum_{i=0}^{\Delta-1} \pi_{i} \sum_{r=0}^{s+\Delta-1-i} p_{r}+\sum_{i=\Delta}^{C} \pi_{i} \sum_{r=0}^{s+\Delta-1} p_{r}\right. \\
& \left.+\sum_{i=C+1}^{s+\Delta-1+C} \pi_{i} \sum_{r=0}^{s+\Delta-1+C-i} p_{r}\right\} .
\end{aligned}
$$

In the following result we show that the optimal value of $s$ can be obtained from the stationary distribution $\Pi$ as a newsvendor critical level.

Proposition 1. The optimal threshold level for a given $\Delta$ is the smallest $s$ that satisfies $\sum_{j=0}^{s+\Delta-1} \pi_{j} \geq \frac{b}{h+b}$.

Proof. We need to show that

$$
\sum_{j=0}^{s+\Delta-1} \pi_{j}=\sum_{i=0}^{\Delta-1} \pi_{i} \sum_{r=0}^{s+\Delta-1-i} p_{r}+\sum_{i=\Delta}^{C} \pi_{i} \sum_{r=0}^{s+\Delta-1} p_{r}+\sum_{i=C+1}^{s+\Delta-1+C} \pi_{i} \sum_{r=0}^{s+\Delta-1+C-i} p_{r} .
$$

By using the balance equations for the stationary distribution, we obtain

$$
\begin{aligned}
\sum_{j=0}^{s+\Delta-1} \pi_{j} & =\sum_{j=0}^{s+\Delta-1} \sum_{i=0}^{\infty} \pi_{i} a_{i, j} \\
& =\sum_{i=0}^{\infty} \pi_{i} \sum_{j=0}^{s+\Delta-1} a_{i, j} .
\end{aligned}
$$

Then,

$$
\begin{aligned}
\sum_{i=0}^{\Delta-1} \pi_{i} \sum_{j=0}^{s+\Delta-1} a_{i, j} & =\sum_{i=0}^{\Delta-1} \pi_{i} \sum_{j=i}^{s+\Delta-1} p_{i-j}=\sum_{i=0}^{\Delta-1} \pi_{i} \sum_{r=0}^{s+\Delta-1-i} p_{r}, \\
\sum_{i=\Delta}^{C} \pi_{i} \sum_{j=0}^{s+\Delta-1} a_{i, j} & =\sum_{i=\Delta}^{C} \pi_{i} \sum_{j=0}^{s+\Delta-1} p_{j}, \text { and } \\
\sum_{i=C+1}^{\infty} \pi_{i} \sum_{j=0}^{s+\Delta-1} a_{i, j} & =\sum_{i=C+1}^{s+\Delta-1+C} \pi_{i} \sum_{j=i-C}^{s+\Delta-1} p_{j+C-i}=\sum_{i=C+1}^{s+\Delta-1+C} \pi_{i} \sum_{r=0}^{s+\Delta-1+C-i} p_{r},
\end{aligned}
$$

and this concludes the proof. 
This newsvendor type result is expected because the threshold level only depends on the holding cost, the backorder cost, and the demand distribution. As a result, if the policy is specified as an $(s, \Delta)$ policy with the threshold level $s$ and a given $\Delta$ value, we calculate $s^{*}(\Delta)$ by using the steady state distribution and unit backorder/holding costs. Finally, the optimal $\Delta$ value, which minimizes the expected cost per period, is determined as follows:

$$
\Delta^{*}=\underset{\Delta \in\{1,2, \ldots, C\}}{\operatorname{argmin}} \mathrm{AC}\left(s^{*}(\Delta), \Delta\right) .
$$

The proposed policy parameter $\Delta$ does not affect the expected cost per period monotonically, as expected. In Fig. 2, we illustrate this relationship.

The following proposition establishes the relationship between $s^{*}(\Delta)$ and $\Delta$.

Proposition 2. Let $s^{*}\left(\Delta^{*}\right)$ be the threshold level for the best $(s, \Delta)$ policy. Then $s^{*}\left(\Delta^{*}\right) \leq s^{*}(1)$, where $s^{*}(1)$ is the optimal threshold level for an $(s, 1)$ policy (the order-up-to-policy).

Proof. Let $\left\{Z_{n}, n=0,1, \ldots\right\}$ be the shortfall process for a $(s, 1)$ policy. Then, $Z_{n+1}=\max \left(D_{n+1}, D_{n+1}+Z_{n}-C\right), n=0,1, \ldots$ Without loss of generality choose $Z_{0}$ and $W_{0}$ in such a way that $W_{0} \leq Z_{0}+\Delta-1$. Suppose that $W_{n} \leq Z_{n}+\Delta-1$ for some $n \geq 0$. Then it follows that

$$
\begin{aligned}
W_{n+1} & \leq \max \left(D_{n+1}+\Delta-1, W_{n}-C+D_{n+1}\right) \\
& \leq \max \left(D_{n+1}+\Delta-1, Z_{n}+\Delta-1-C+D_{n+1}\right) \\
& =\Delta-1+\max \left(D_{n+1}, Z_{n}-C+D_{n+1}\right) \\
& =\Delta-1+Z_{n+1} .
\end{aligned}
$$

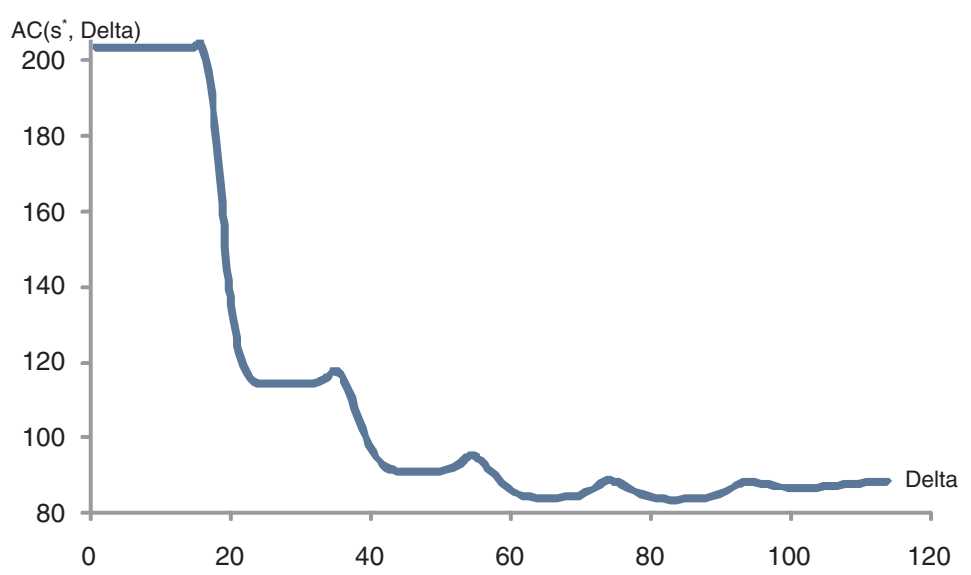

Fig. 2. $\Delta$ versus expected cost per period with optimal threshold level, $s^{*}$. 
The first inequality follows from the definition of $W_{n+1}$, and the second inequality is due to the induction argument. Therefore, $W_{n} \leq Z_{n}+\Delta-1$ for all $n=0,1, \ldots$ Let $W$ and $Z$ be the limit random variables for $W_{n}$ and $Z_{n}$, respectively. Then,

$$
\operatorname{Pr}\{W \leq s+\Delta-1\} \geq \operatorname{Pr}\{Z+\Delta-1 \leq s+\Delta-1\}=\operatorname{Pr}\{Z \leq s\} .
$$

Hence, $\operatorname{Pr}\{Z \leq s\} \geq b /(h+b)$ implies that $\operatorname{Pr}\{W \leq s+\Delta-1\} \geq b /(h+b)$ and therefore $s^{*}(\Delta) \leq s^{*}(1)$ for any $\Delta \in\{1,2, \ldots, C\}$ and the claim follows.

\subsection{Testing the performance of the heuristic}

We construct a DTMC for the infinite horizon problem and determine an optimal threshold value using the steady state probabilities. In this section, we apply (1) the $(s, \Delta)$ policy, (2) its more restricted version, the all-or-nothing policy (where $\Delta=C$ ), and finally (3) the modified base stock policy (where $\Delta=1$ ) as heuristics for the problem and test the performance of these heuristics on a set of instances. In generating these instances, we use different combinations of problem parameters such as demand distribution, cost parameters, and capacity values. For each setting, we compute the average cost of the heuristics by simulating all three policies with respective stationary threshold values. That is, we assume that a fixed policy for every period and solve the underlying DTMC to obtain the policy parameters and use these parameters to simulate the policies. Then, we compare the results of the heuristics with the result of the DP model. We employ the algorithm below to determine the best $(s, \Delta)$ policy. Note that $\Delta=1$ corresponds to modified base stock policy, $\Delta=C$ corresponds to all-or nothing policy.

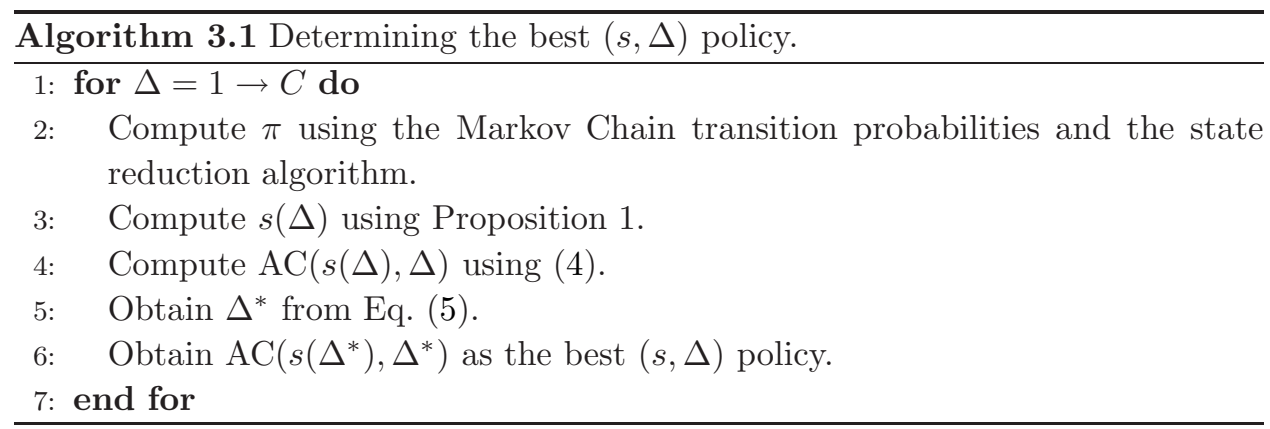

We need to find the optimal solution via a DP model so to benchmark the performance of the heuristics mentioned above. The main handicap of DP is that as the number of periods-to-go increases, the number of possible initial inventory values increases rapidly. If we could solve the dynamic program for sufficiently large planning horizons, the average cost per period would converge and would be independent of the initial inventory value at the beginning of the planning horizon. However, this is not the case and the average cost values cannot recover from the 
dependence on the initial inventory value with the planning horizon that we consider (1,000 periods) and thus remain a function of the initial inventory value.

Therefore, we consider all initial inventory levels between $[\mu-3 \sigma, \mu+3 \sigma]$ where $\mu$ is the expected value of the demand and $\sigma$ is the standard deviation of the demand. The maximum percentage deviation of the average cost of a heuristic from the optimal average cost calculated by the DP model, $\theta$, is calculated as:

$$
\theta=\max _{x \in[\mu-3 \sigma, \mu+3 \sigma]} \frac{\tilde{A}_{n}(x)-A_{n}(x)}{A_{n}(x)} \times 100,
$$

where $A_{n}(x)$ is the expected cost per period for an $n$-period problem solved by DP, given that the beginning inventory position is $x$ and $\tilde{A}_{n}(x)$ is the expected cost per period of a heuristic over the same planning horizon. We run a terminating simulation for the same number of periods and the same initial inventory value as the DP to calculate $\tilde{A}_{n}(x)$. Note that the maximum percentage deviation is calculated over the interval $[\mu-3 \sigma, \mu+3 \sigma]$. If this maximum value occurs at a boundary point, $\mu-3 \sigma$ or $\mu+3 \sigma$, we extend the interval by $\sigma$ and recalculate the maximum percentage deviation value. We use this deviation value as a key performance indicator in testing the performance of the heuristics.

\section{Computational Study}

In order to evaluate the performance of the heuristics more clearly, we perform two different computational analyses. In the first analysis, we choose only eight sets of demand distribution with certain characteristics and analyze the performance of all heuristics on each demand set individually over 1,536 instances. Our motivation of this analysis is the fact that the optimal policy is significantly affected by the demand distribution and in an aggregated result it is quite difficult to observe this effect. Therefore, we choose representative demand distributions to mimic the normal, uniform and Poisson type demand distributions, which are widely used in analyzing inventory systems as well as a restrictive demand distribution similar to a deterministic demand, which we observe to be the most challenging demand distribution in this context. From this analysis, we gather valuable insights on the policy and setting compatibility and the dominance of $(s, \Delta)$ policy over the other two. The analysis might seem too restrictive as we specify the demand distributions even though we experiment with different distributions. To this end, we perform a second computational analysis where we randomly generate 100 sets of demand distribution of different characteristics. This is an extensive analysis conducted over 12,000 instances, which provides an unbiased overall evaluation of the three heuristics.

For the first analysis, we itemize the parameters used to generate different problem settings as follows:

- Demand Distribution: We experiment with eight sets of demand distribution, all of which have an expected value of 19.05. For each demand set, we present the possible demand values, their respective probabilities and the coefficient of 
Table 1. Demand distributions.

\begin{tabular}{cccc}
\hline Dem. set & Possible dem. values & Probabilities & CoV \\
\hline 1 & $15,16,17,18,19$ & $0.03,0.07,0.1,0.165,0.24$, & 0.097 \\
& $20,21,22,23$ & $0.175,0.12,0.07,0.03$ & 0.011 \\
2 & 19,20 & $0.95,0.05$ & 0.655 \\
3 & $0,27,28,30$ & $0.3,0.61,0.06,0.03$ & 0.351 \\
4 & $0,5,11,20,26,29$ & $0.01,0.03,0.26,0.39,0.25,0.06$ & 0.614 \\
5 & $0,14,15,35,36$ & $0.12,0.29,0.29,0.16,0.14$ & 1.046 \\
6 & $0,1,40$ & $0.475,0.05,0.475$ & 0.136 \\
7 & $15,16,17,18,19$ & 0.1114 for all & \\
& $20,21,22,23$ & & \\
8 & $12,13,14,1516,17,18$ & $0.026,0.038,0.052,0.066,0.078,0.088,0.093$ & 0.208 \\
& $19,20,21,22,23,24,25$ & $0.093,0.089,0.08,0.069,0.058,0.046,0.035$ & \\
& $26,27,28,29,30,31,32$ & $0.026,0.018,0.012,0.008,0.005,0.003,0.002$ & \\
\hline
\end{tabular}

variation values in Table 1 . The first set resembles the normal distribution with several possible demand values whereas the second set is similar to the deterministic demand and so consists of two possible demand values. The third set has a high probability of having no demand in a period. The fourth set resembles the normal distribution with higher standard deviation compared to the first demand set. The fifth set has a bimodal type distribution. The sixth set has the highest standard deviation. The seventh set resembles the uniform distribution with several possible demand values and finally, the eighth set resembles a truncated Poisson distribution. For these eight sets of demand distributions, the coefficient of variation varies between 0.011 and 1.046, respectively, which does not seem to affect the performance of the heuristics. As will be discussed later, discreteness of the demand distribution is likely to have an effect on the optimal ordering structure, hence on the performance of the heuristics.

- Cost Parameters: We take the holding cost constant, $h=1$, in our experiments and relatively set the other cost parameters. The variable cost does not affect the optimal policy, hence we take $v=0$. The remaining cost parameters that we use in our computations are as follows: $b \in\{3,5,10,20\}$ and $K \in\{10,25,50,100,200,500\}$

- Capacity: The ratio of the expected demand in a period to the capacity value is equal to the utilization of the capacity in the long run. We select the capacity values in our experiments so that the capacity utilization values are very high (such as $95 \%, 90 \%, 85 \%$, which corresponds to capacity-wise challenging instances), or normal (such as 75\%,65\%,50\%) and finally very low (such as 25\%, 15\%, which corresponds to capacity-wise relaxed instances). Due to the integrality requirement of the capacity value, the resulting capacity utilization values turn out be as follows: $95 \%, 90 \%, 86 \%, 76 \%, 65 \%, 50 \%, 25 \%$, and $17 \%$. 
We present the results for the first demand set in Table 2 and present the results for the remaining demand sets in Appendix A. In Table 2, the column "95\%-AVE" presents the average $\theta$ value over the instances where the capacity utilization is $95 \%$. Similarly, the column "95\%-MAX" presents the maximum $\theta$ value the instances where the capacity utilization is $95 \%$. The rest is similar. The first part of Table 2 presents the results for the best $(s, \Delta)$ policy, the second part presents the results for the all-or-nothing policy and finally the last part presents the results for the modified base stock policy. For example, the first row of Table 2 presents the average and maximum $\theta$ value over all the instances with a fixed ordering cost of 10 . Note that, the best $(s, \Delta)$ policy cannot be worse than the all-or-nothing policy, as setting $\Delta=C$ in the $(s, \Delta)$ policy leads to the all-or-nothing case. Having said that, our computational results show that the performance of the best $(s, \Delta)$ policy is significantly superior to the all-or-nothing policy and the modified base stock policy for almost all the parameter settings that we considered. In a few settings (that we present below) the best $(s, \Delta)$ policy occurs at $\Delta=C$.

We observe from the result in Table 2 that as the fixed ordering cost increases, the performance of the all-or-nothing heuristic improves, which is in line with our expectations. On the other hand, the performance of the modified base stock heuristic deteriorates as the fixed cost increases. Also from Table 2, we see that as the capacity utilization increases, the performance of both the all-or-nothing heuristic and modified base stock heuristic improves. For the $(s, \Delta)$ heuristic, we do not observe a clear pattern with respect to fixed ordering cost or capacity utilization, however, the deviation results are almost equal to zero. Thus, the performance of $(s, \Delta)$ heuristic is robust and consistently good with respect to all the parameters in this demand setting.

Similar observations can be made for other distributions. For details, see the results in Appendix A.

We summarize the results of the computational analysis in Table 3 . The first row of Table 3 presents the average and maximum deviations of the best $(s, \Delta)$ policy, the all-or-nothing policy and the modified base stock policy over all 1,536 instances. In the next eight rows, the average and maximum deviations with respect to different demand distributions are given. Similarly, the average and maximum deviations are presented with respect to different fixed cost values in rows 10-15, with respect to different capacity utilization values in rows 16-23.

We observe that the average percentage deviation of the best $(s, \Delta)$ policy from the DP cost figures is $0.36 \%$, whereas the maximum deviation is $4.88 \%$. On the other hand, we observe that the average percentage deviation of the all-or-nothing policy from the DP cost figures is $18.26 \%$, whereas the maximum deviation is $414.33 \%$. Finally, we observe that the average percentage deviation of the modified base stock policy from the DP cost figures is $44.97 \%$, whereas the maximum deviation is $369.83 \%$. With respect to different demand distributions, the performance of best $(s, \Delta)$ policy is consistently good, on the other hand the performances of all-ornothing heuristic and modified base stock heuristic are not only worse compared 


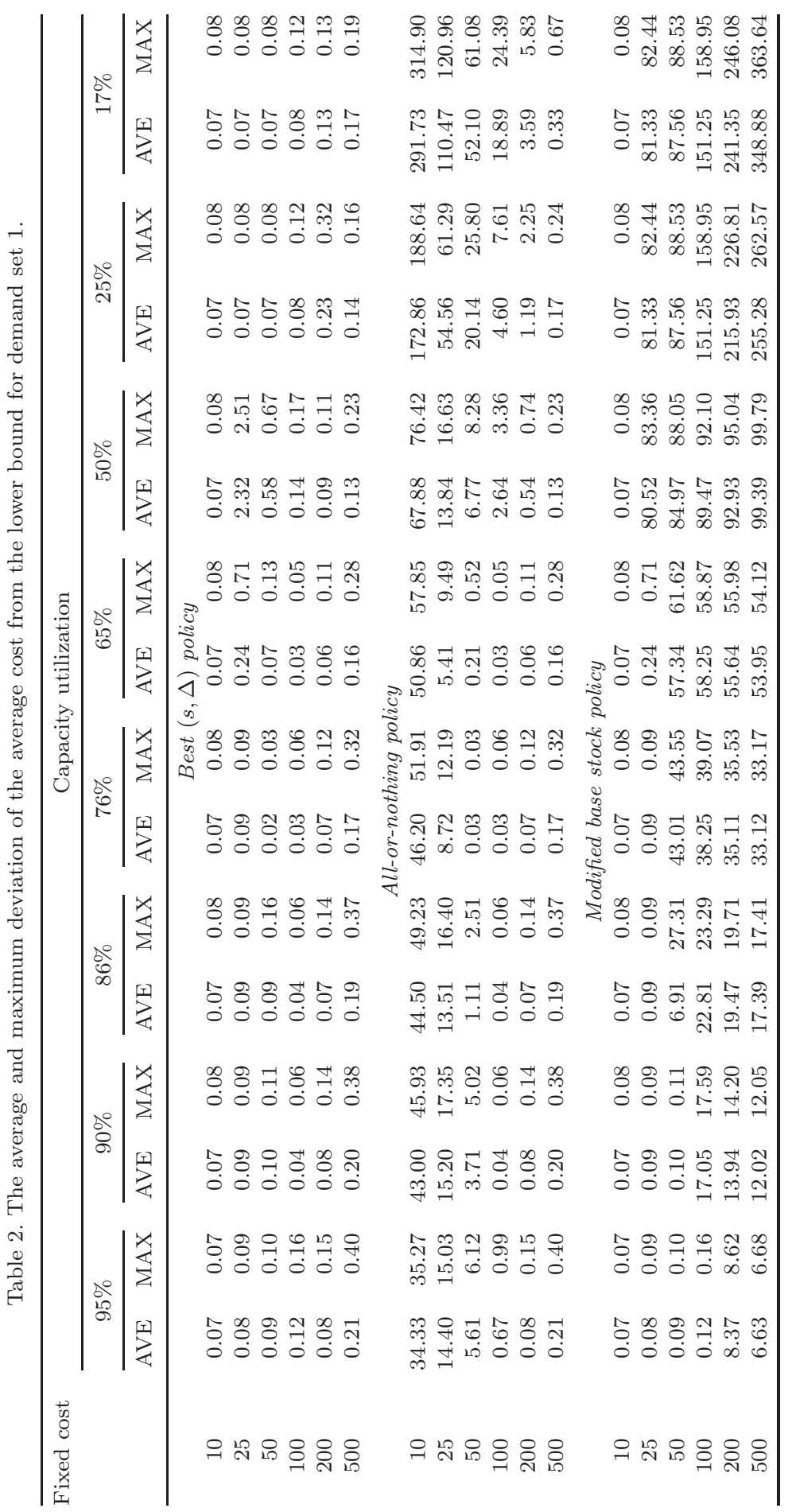


Table 3. Summary of results 1 - Best $(s, \Delta)$ policy, all-or-nothing policy and modified base stock policy.

\begin{tabular}{|c|c|c|c|c|c|c|c|}
\hline & & \multicolumn{2}{|c|}{ Best $(s, \Delta)$} & \multicolumn{2}{|c|}{ All-or-not. } & \multicolumn{2}{|c|}{ Mod. base sto. } \\
\hline & & AVE & MAX & AVE & MAX & AVE & MAX \\
\hline \multicolumn{2}{|l|}{ Total } & 0.36 & 4.88 & 18.26 & 414.33 & 44.97 & 369.83 \\
\hline \multirow[t]{8}{*}{ Demand } & Set1 & 0.16 & 2.51 & 23.15 & 314.90 & 55.20 & 363.64 \\
\hline & Set2 & 0.25 & 3.49 & 34.84 & 414.33 & 58.24 & 362.12 \\
\hline & Set3 & 0.50 & 4.35 & 20.52 & 266.35 & 26.77 & 230.06 \\
\hline & Set4 & 0.13 & 1.17 & 11.91 & 199.53 & 51.71 & 357.72 \\
\hline & Set5 & 0.39 & 3.68 & 8.53 & 156.73 & 38.96 & 309.10 \\
\hline & Set6 & 0.75 & 3.50 & 13.15 & 182.38 & 17.03 & 158.08 \\
\hline & Set7 & 0.22 & 2.20 & 20.50 & 304.72 & 55.07 & 365.41 \\
\hline & Set8 & 0.48 & 4.88 & 13.49 & 208.89 & 56.77 & 369.83 \\
\hline \multirow[t]{6}{*}{ Fixed cost } & 10 & 0.39 & 4.88 & 68.79 & 414.33 & 0.89 & 10.25 \\
\hline & 25 & 0.49 & 4.12 & 24.93 & 156.31 & 20.16 & 102.01 \\
\hline & 50 & 0.32 & 3.84 & 10.67 & 77.72 & 36.91 & 117.47 \\
\hline & 100 & 0.30 & 3.40 & 3.64 & 35.70 & 53.47 & 170.72 \\
\hline & 200 & 0.32 & 3.59 & 1.09 & 18.34 & 70.86 & 257.54 \\
\hline & 500 & 0.34 & 2.42 & 0.45 & 4.86 & 87.53 & 369.83 \\
\hline \multirow{8}{*}{$\begin{array}{l}\text { Capacity } \\
\text { utilization }\end{array}$} & $95 \%$ & 1.22 & 4.88 & 5.51 & 77.01 & 4.06 & 13.57 \\
\hline & $90 \%$ & 0.41 & 2.26 & 5.24 & 77.16 & 6.32 & 19.48 \\
\hline & $86 \%$ & 0.24 & 1.16 & 5.20 & 77.73 & 9.38 & 27.68 \\
\hline & $76 \%$ & 0.17 & 2.08 & 5.60 & 80.62 & 20.05 & 46.87 \\
\hline & $65 \%$ & 0.15 & 2.29 & 8.44 & 87.92 & 29.73 & 64.56 \\
\hline & $50 \%$ & 0.32 & 3.49 & 13.30 & 116.46 & 56.63 & 102.01 \\
\hline & $25 \%$ & 0.23 & 3.59 & 35.99 & 257.65 & 107.67 & 268.36 \\
\hline & $17 \%$ & 0.13 & 2.42 & 66.82 & 414.33 & 125.90 & 369.83 \\
\hline
\end{tabular}

to best $(s, \Delta)$ policy for all demand sets but also inconsistent in different demand distributions. Note that there seems to be no effect of the value of the coefficient of variation on the results observed. We believe that the effect of discreteness, as well as the fact that there is only a limited number of demand values contributes more to the result.

All the computations take under a minute meaning both simulating the DP and the other polices. However, the real difficulty in computations is the excessive memory requirements in simulating DP, which is due to the planning horizon and the parameters such as capacity and possible demand values. This is the reason for not generating a demand distribution with a longer tail. Also, we might not be able to use DP for longer time horizons due to this memory restriction.

Next, we test the performance of the heuristics over randomly generated demand. We generate 100 sets of demand distribution, all of which have an expected value of 25 . The demand sets generated are selected in equal number from each of the following four distributions: Demand sets demand sets with a few (2-4) possible demand values, demand sets resembling uniform distribution, demand sets resembling normal distribution, demand sets resembling Poisson distribution. The last three types of demand sets have a minimum of 7 and maximum of 15 possible 
nonzero demand values all between a lower and an upper bound so as to limit the tails of the generated distributions. The uniform and normal type demand distributions have specific intervals between the lower and the upper bound where all integers correspond to realizable demand values. The probabilities are generated using the respective demand distribution type and normalized so that the expected value is equal to 25 . The Poisson type distributions have randomly generated realizable values between the lower and the upper bound, the corresponding probabilities are computed using a Poisson distribution p.m.f. with a mean value of 25 and finally normalized so that the expected value is equal to 25 . We use the same parameter sets for backorder cost and fixed cost. For these hundred sets of demand distributions, the average of coefficient of variation values is equal to 0.547436 whereas the maximum value is equal to 0.934067 . As for the capacity values, we select the capacity parameter so that the capacity utilization values are $89.3 \%, 76 \%, 50 \%$, $33.3 \%$, and $25 \%$.

We summarize the results of the second computational analysis in Table 4. The first row of Table 4 presents the average and maximum deviations of the best $(s, \Delta)$ policy, the all-or-nothing policy and the modified base stock policy over all 12,000 instances. In the next four rows, the average and maximum deviations with respect to different demand distribution sets, respectively almost deterministic discrete, uniform, normal, and Poisson distributions, are given. Similarly, the average and maximum deviations are presented with respect to different fixed cost values in rows 6-11, with respect to different capacity utilization values in rows 12-16. We observe results similar to the first computational study. The average percentage deviation

Table 4. Summary of results 2 - Best $(s, \Delta)$ policy, all-or-nothing policy and modified base stock policy.

\begin{tabular}{|c|c|c|c|c|c|c|c|}
\hline & & \multicolumn{2}{|c|}{ Best $(s, \Delta)$} & \multicolumn{2}{|c|}{ All-or-not. } & \multicolumn{2}{|c|}{ Mod. base sto. } \\
\hline & & AVE & MAX & AVE & MAX & AVE & MAX \\
\hline \multicolumn{2}{|l|}{ Total } & 0.14 & 8.15 & 18.20 & 467.26 & 43.21 & 251.60 \\
\hline \multirow{4}{*}{ Demand } & Set1 & 0.22 & 8.15 & 28.56 & 467.26 & 35.18 & 244.51 \\
\hline & Set2 & 0.12 & 1.53 & 11.61 & 171.78 & 41.59 & 246.63 \\
\hline & Set3 & 0.11 & 1.47 & 12.74 & 388.93 & 42.87 & 241.54 \\
\hline & Set 4 & 0.12 & 6.02 & 19.89 & 229.45 & 53.22 & 251.60 \\
\hline \multirow[t]{6}{*}{ Fixed cost } & 10 & 0.09 & 1.44 & 63.18 & 467.26 & 1.11 & 11.54 \\
\hline & 25 & 0.09 & 2.64 & 28.39 & 369.76 & 4.75 & 82.81 \\
\hline & 50 & 0.20 & 8.15 & 11.22 & 332.49 & 29.87 & 92.32 \\
\hline & 100 & 0.18 & 4.22 & 3.95 & 276.70 & 57.65 & 148.94 \\
\hline & 200 & 0.14 & 2.51 & 1.66 & 207.18 & 76.39 & 210.42 \\
\hline & 500 & 0.16 & 2.11 & 0.81 & 118.13 & 89.52 & 251.60 \\
\hline \multirow{5}{*}{$\begin{array}{l}\text { Capacity } \\
\text { utilization }\end{array}$} & $89 \%$ & 0.31 & 1.44 & 9.50 & 467.26 & 5.48 & 21.71 \\
\hline & $76 \%$ & 0.08 & 1.62 & 6.86 & 283.41 & 14.93 & 45.12 \\
\hline & $50 \%$ & 0.15 & 8.15 & 15.18 & 289.66 & 41.06 & 98.85 \\
\hline & $33 \%$ & 0.12 & 4.22 & 23.36 & 249.12 & 69.53 & 184.08 \\
\hline & $25 \%$ & 0.06 & 2.11 & 36.10 & 346.49 & 85.07 & 251.60 \\
\hline
\end{tabular}


of the best $(s, \Delta)$ policy from the DP cost figures is $0.14 \%$, whereas the maximum deviation is $8.15 \%$. The performance of the best $(s, \Delta)$ policy is consistently good under all possible combinations of parameters and the average deviation value is very close to zero. On the other hand, we observe that the average percentage deviation of the all-or-nothing policy from the DP cost figures is $18.2 \%$, whereas the maximum deviation is $467.26 \%$. Finally, we observe that the average percentage deviation of the modified base stock policy from the DP cost figures is $43.21 \%$, whereas the maximum deviation is $251.6 \%$. Again, all the computations take under a minute.

As a conclusion, for the infinite horizon problem with capacity constraint and the fixed cost, the $(s, \Delta)$ policy with a threshold level is a well-performing heuristic under all combinations of problem parameters, even in problematic demand structures. This policy significantly outperforms both the all-or-nothing policy and the modified base stock policy.

\section{Conclusion}

The optimal ordering policy for the stationary, single-item, multi-period inventory problem under capacity constraints has not been completely characterized. There exist some attempts to partially characterize the optimal policy, however the nonmonotonic characteristic of the ordering quantity as a function of inventory position complicates the problem.

In this study, we construct a DTMC for the problem with stationary parameters and an arbitrary discrete demand distribution under $(s, \Delta)$ policy. We define the states of the Markov chain as the shortfall levels from the order-up-to-level. The optimal threshold level turns out to be the point that satisfies the critical ratio of $\frac{b}{b+h}$, as in the base stock policy. For this problem, we show that the optimal policy is not a modified $(s, S)$-type policy, so the application basically is a heuristic.

To test the performance of the heuristic, we compare the average cost per period through DP that finds a lower bound on the optimal solution, then we compare the results with the ones we obtain from the DTMC model. The computational analysis reveals that application of the best $(s, \Delta)$ policy as a heuristic to the problem yields satisfactory results, significantly better than the all-or-nothing policy and the modified base stock policy.

\section{Appendix A}

We present the result for the second demand set in Table A.1. Recall that in the second demand set there are only two possible demand values. In Sec. 2.2, we illustrate with an example that this type of demand distribution results in a more kinky order quantity function and hence presents a relatively more challenging instance. Therefore, we expect the performance of the heuristics to be slightly lower compared to the performance on the first set of instances. From the results in Table A.1, we observe that this is in fact the case and the average deviation and the maximum 


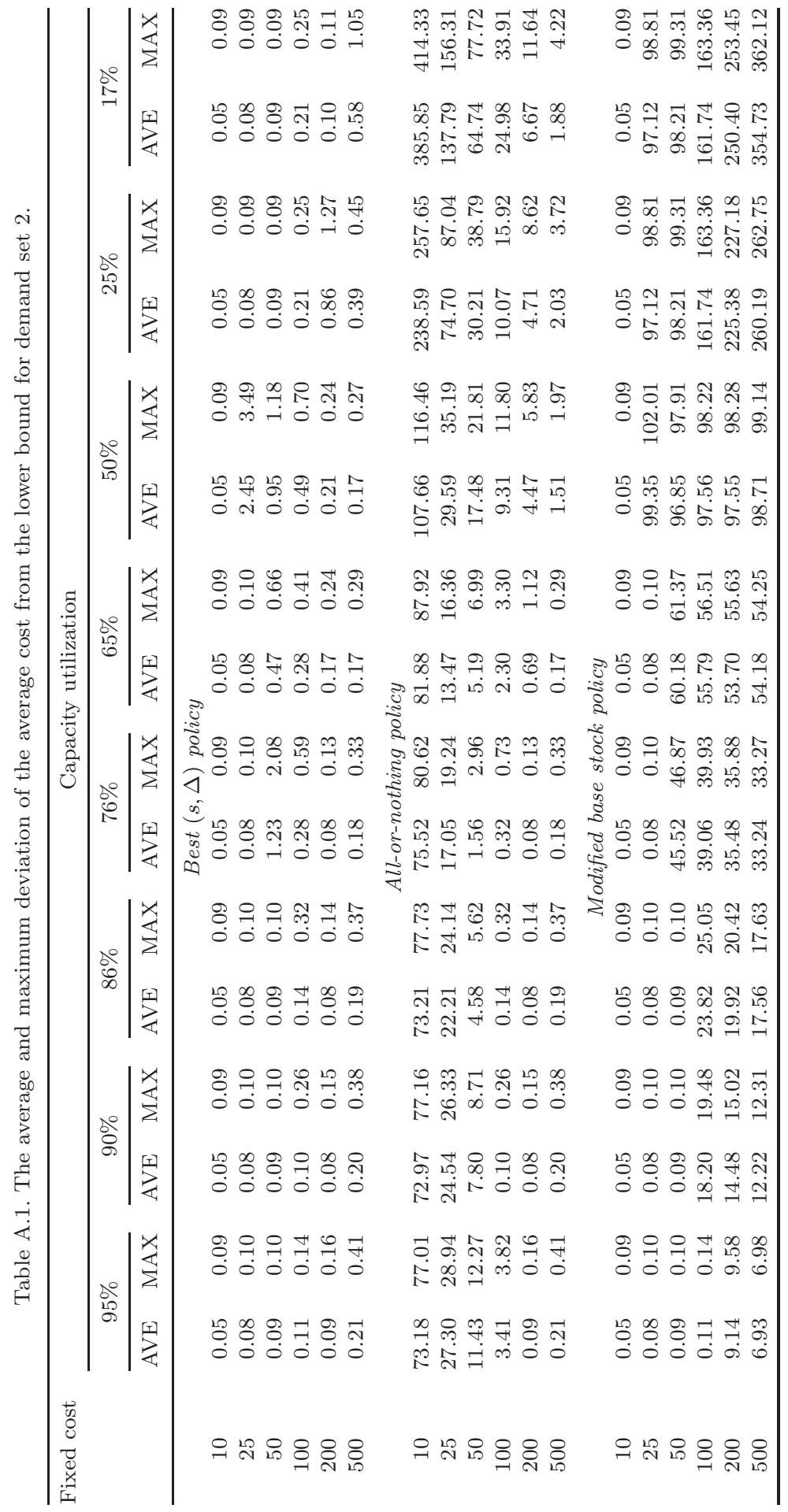




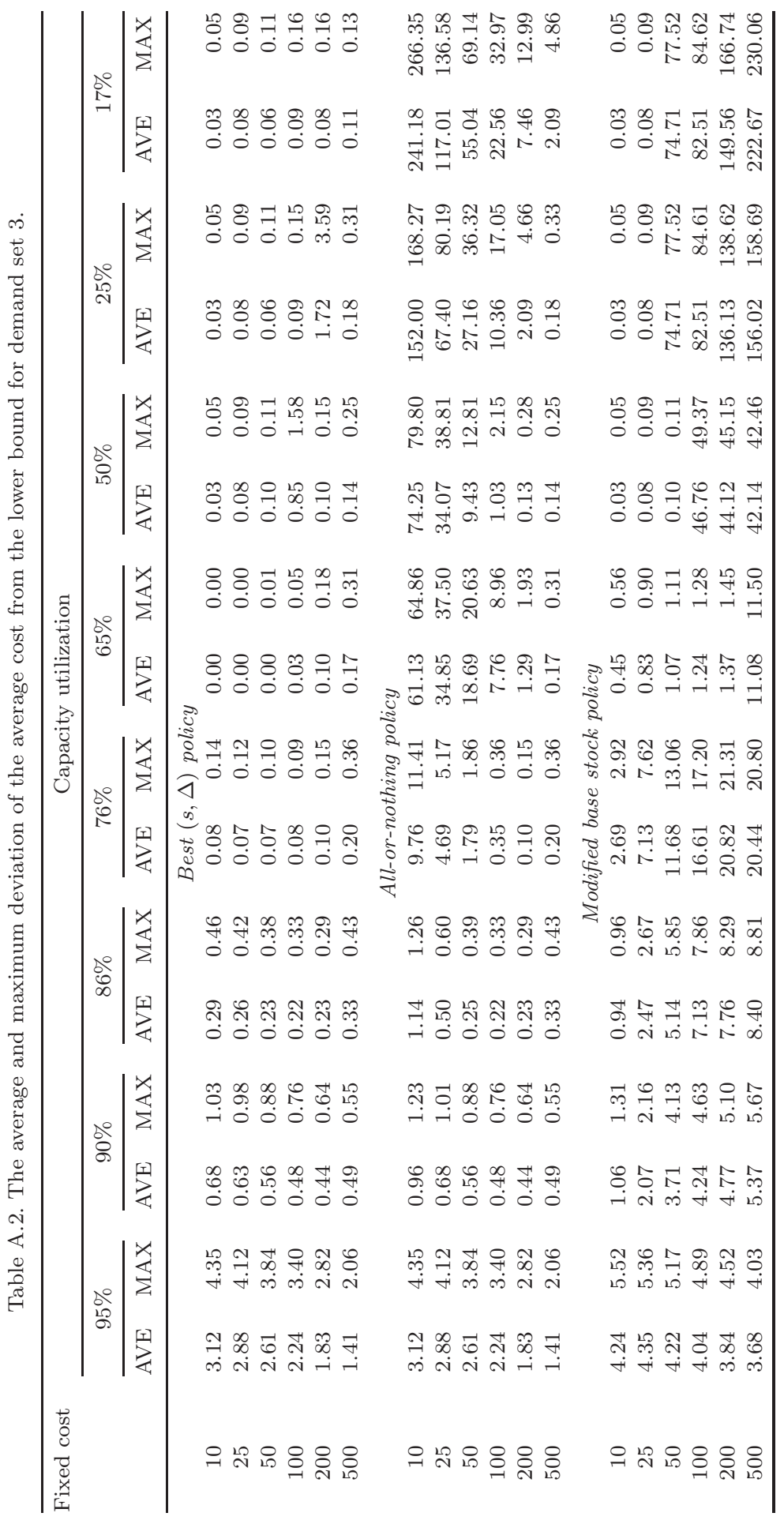




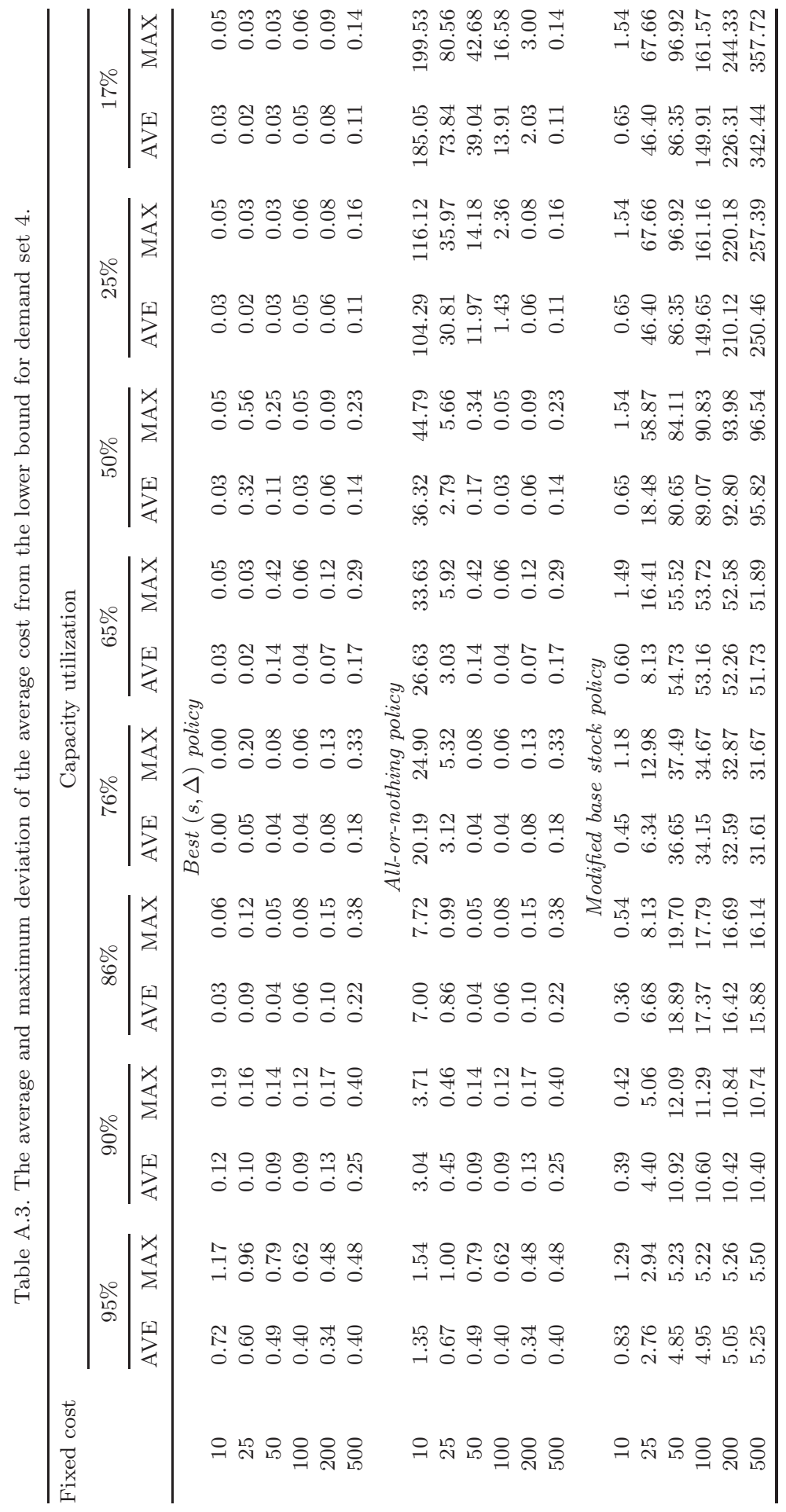




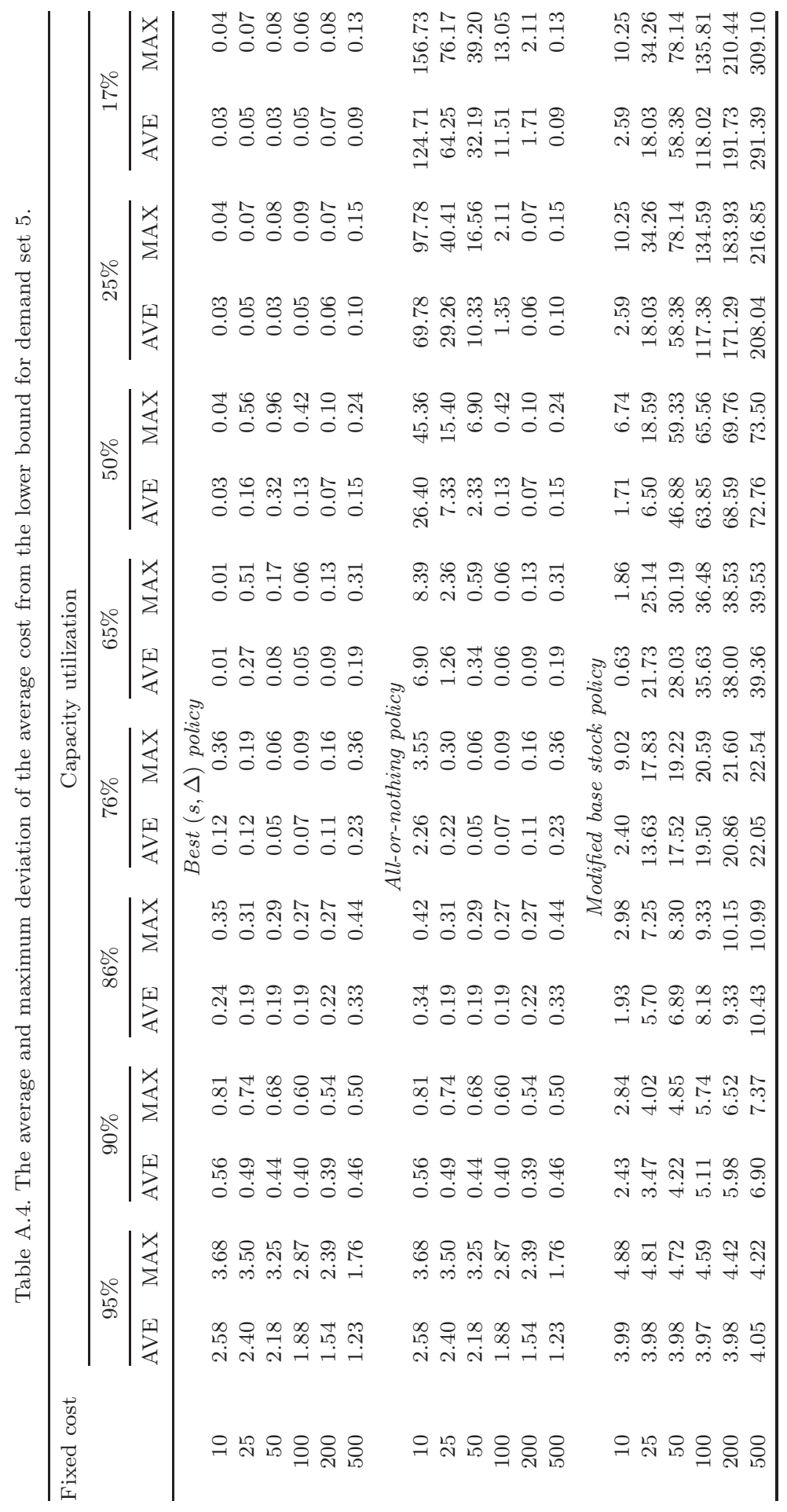




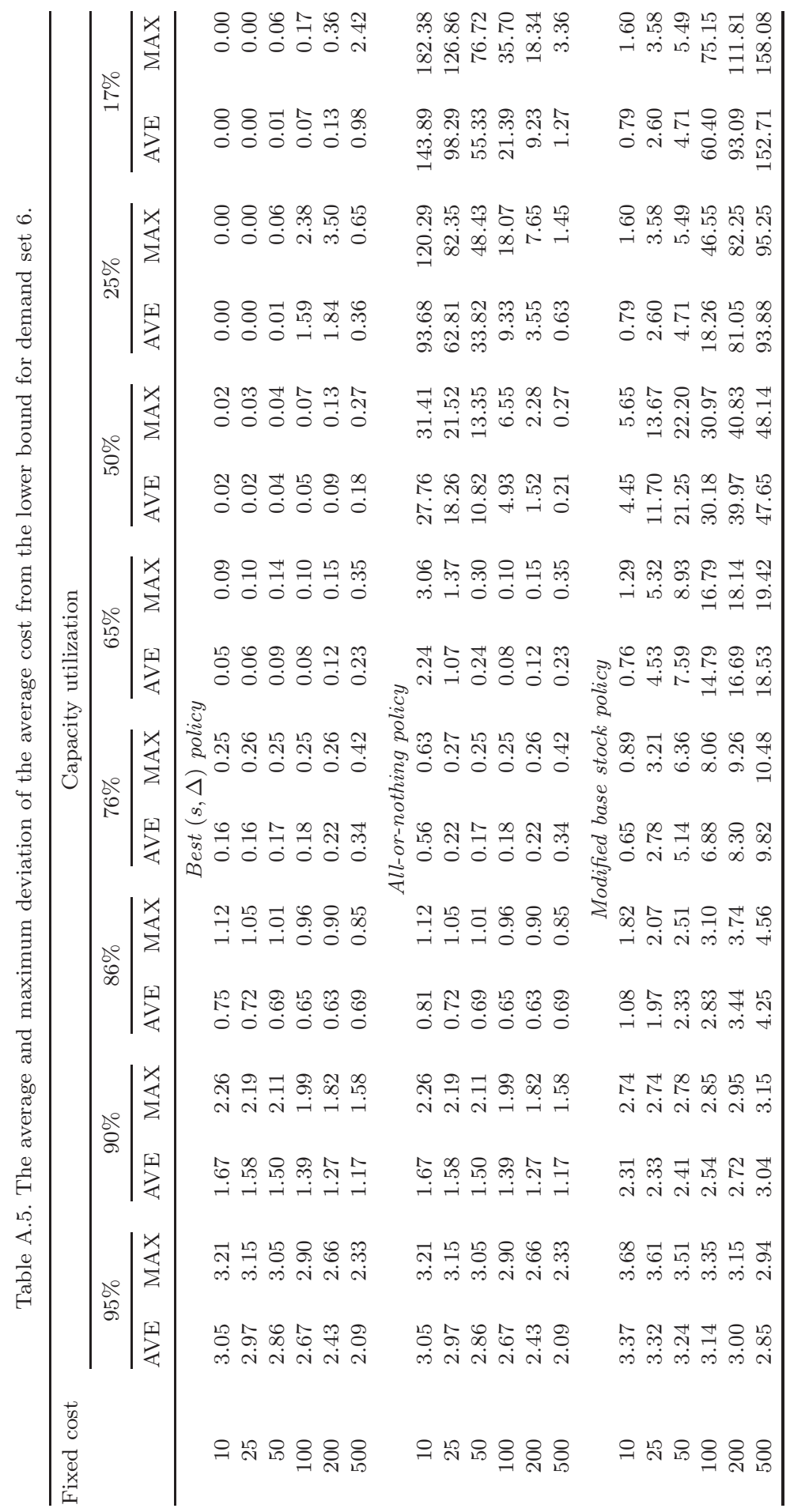




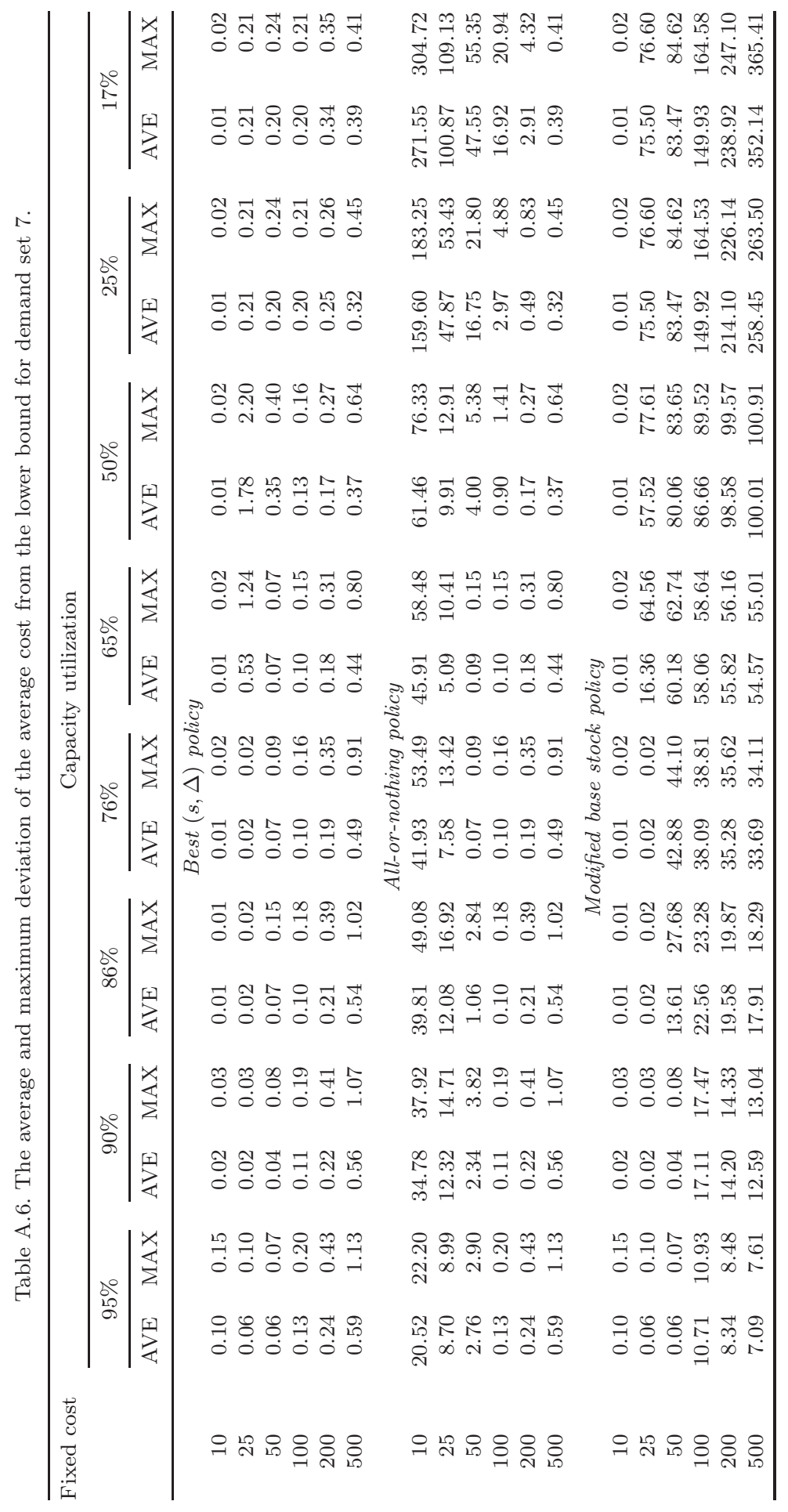




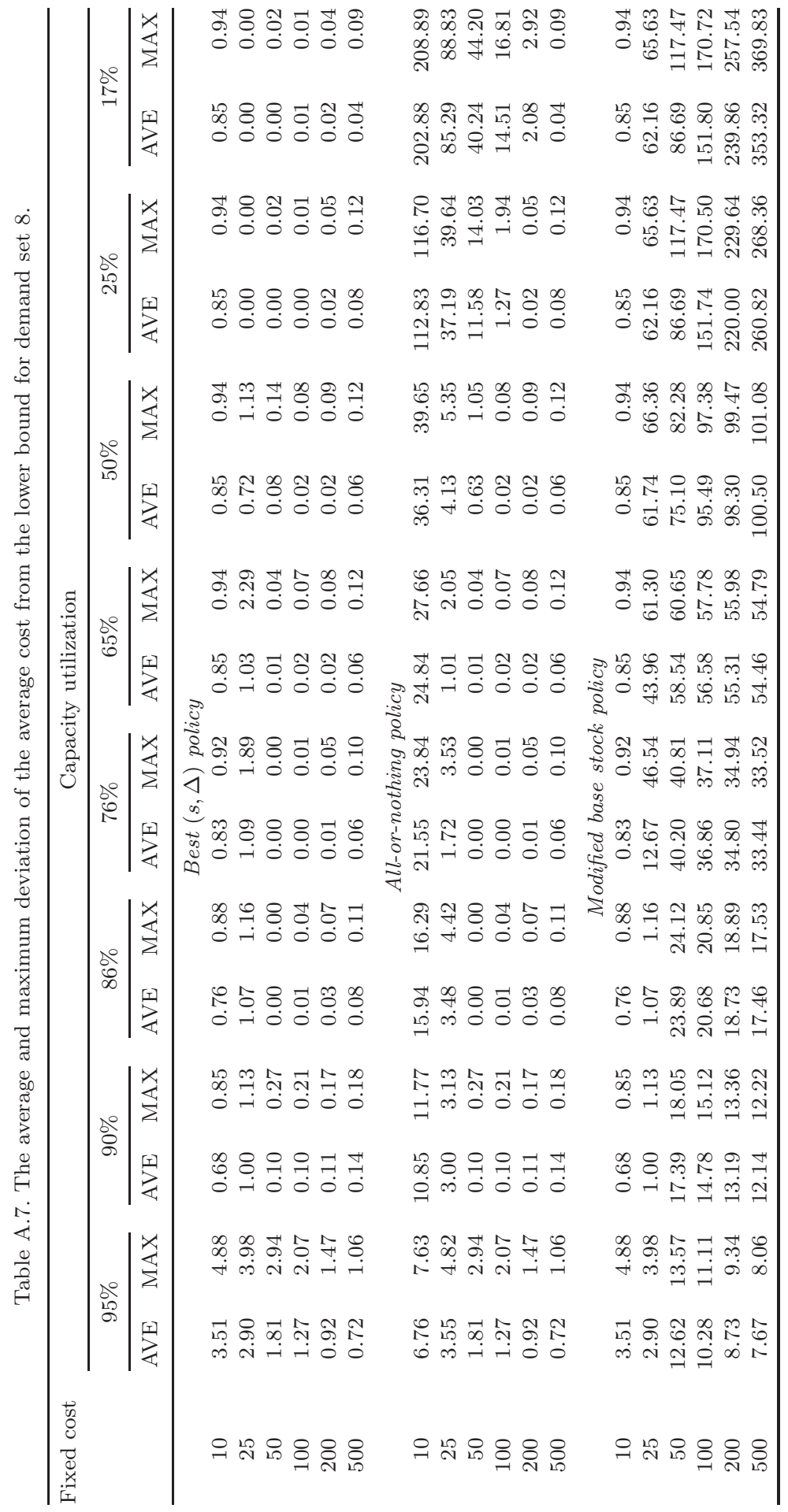


deviation values for all the heuristics are greater than corresponding values of the previous set of instances except a few exceptions. However, the deviation values for the best $(s, \Delta)$ policy are still very close to zero.

We present the result for the third demand set in Table A.2. The significance of this demand set is that the probability of having no demand in any period is high, $30 \%$, which seems to affect the performance of the best $(s, \Delta)$ policy and the modified base stock policy when the capacity utilization is high.

We present the result for the fourth demand set in Table A.3. This demand set is a discretized version of a normal distribution and has a higher standard deviation compared to the first demand set. Interestingly, the performances of the heuristics do not diminish as the standard deviation of the demand increases.

We present the result for the fifth demand set in Table A.4. This demand set has a bimodal distribution and some of the demand values are much higher than the expected value. This again affects the performance of the best $(s, \Delta)$ policy and the modified base stock policy when the capacity utilization is high.

We present the result for the sixth demand set in Table A.5. This demand set has the highest standard deviation value, which again affects the performance of the best $(s, \Delta)$ policy and the modified base stock policy when the capacity utilization is high.

We present the results for the seventh demand set, which resembles the uniform distribution, and the eighth demand set, which resembles the Poisson distribution, in Tables A.6 and A.7 respectively. Based on the results, we first observe that Poisson type demand distribution presents a higher challenge compared to uniform type demand distribution and next we observe that the performance of the best $(s, \Delta)$ policy is superior to the others similar to the previous demand sets.

\section{References}

Chan, GH and Y Song (2003). A dynamic analysis of the single-item periodic stochastic inventory system with order capacity. European Journal of Operational Research, 146, 529-542.

Federgruen, A and P Zipkin (1986). An inventory model with limited production capacity and uncertain demands II. The discounted-cost criterion. Mathematics of Operations Research, 11(2), 208-215.

Gallego, G and A Scheller-Wolf (2000). Capacitated inventory problems with fixed order costs: Some optimal policy structure. European Journal of Operational Research, $126,603-613$.

Gallego, G and LB Toktay (2004). All-or-nothing ordering under a capacity constraint. Operations Research, 52(6), 1001-1002.

Girlich, HJ and A Chikán (2001). The origins of dynamic inventory modelling under uncertainty: (the men, their work and connection with the Stanford Studies). International Journal of Production Economics, 71(13), 351-363.

Glynn, JE (1989). A discrete-time storage process with a general release rule. Journal of Applied Probability, 26(3), 566-583.

Heyman, DP and MJ Sobel (1991). Stochastic Models in Operations Research, Vol. I. Amsterdam, The Netherlands: Elsevier Science Publishers. 
Ozer, O and W Wei (2004). Inventory control with limited capacity and advance demand information. Operations Research, 52(6), 988-1000.

Scarf, HE (1960). The optimality of $(s, S)$ policies in the dynamic inventory problem. In Mathematical Models in the Social Sciences, Stanford, CA: Stanford University Press.

Shaoxiang, C (2004). The infinite horizon periodic review problem with setup costs and capacity constraints: A partial characterization of the optimal policy. Operations Research, 52(3), 409-421.

Shaoxiang, C and M Lambrecht (1996). X-Y band and modified $(s, S)$ policy. Operations Research, 44(6), 1013-1019.

Wijngaard, J and ND van Foreest (2010). On the optimal policy for the single-product inventory problem with set-up cost and a restricted production capacity. Technical Report, University of Groningen, Research Institute SOM (Systems, Organisations and Management).

Wjingaard, J (1972). An inventory problem with constraint order capacity. Technical Report, Eindhoven University of Technology.

Okan Örsan Özener is an Assistant Professor in the Industrial Engineering Department, Ozyegin University. He received his BS and MS degrees in Industrial Engineering from the Middle East Technical University and MS in Operations Research and $\mathrm{PhD}$ in Industrial and Systems Engineering from Georgia Institute of Technology. His research interests are in integer/combinatorial optimization and non-cooperative/cooperative game theory and their applications in supply chain management primarily on transportation, distribution, and logistics systems planning and control.

Refik Güllü is a Professor in the Industrial Engineering Department of Bogazici University, Istanbul, Turkey. He received his BS and MS degrees in Industrial Engineering from the Middle East Technical University, Ankara, Turkey, and MS and $\mathrm{PhD}$ degrees in Operations Research from the School of ORIE, Cornell University, New York. Dr. Güllü worked at Middle East Technical University as a faculty member before joining Bogazici University. Dr. Güllü's main research interests are stochastic modeling of production/inventory systems, queueing theory, and supply uncertainty problems.

Nesim Erkip is a Professor of Industrial Engineering at Bilkent University, Ankara, Turkey. He received his BS degree in Industrial Engineering from the Middle East Technical University, Ankara, Turkey, and MSc and PhD degrees in IE\&EM from Stanford University. Prof. Erkip worked at Middle East Technical University as a faculty member before joining Bilkent University, and visited Cornell University, University of California at Berkeley, Eindhoven Technical University, and NYU. His main research interests are in multi-echelon inventory theory, issues in supply chain management, and operations management. 